\title{
Comparative study of adjuvant chemotherapeutic efficacy of docetaxel plus cyclophosphamide and doxorubicin plus cyclophosphamide in female
} breast cancer

\author{
Muhammad Adeel' \\ Muhammad Asif' \\ Muhammad Naeem Faisal ${ }^{2}$ \\ Muhammad Hasanain \\ Chaudary $^{3}$ \\ Muhammad Sheraz Malik ${ }^{4}$ \\ Muhammad Khalid \\ 'Department of Computer Science, \\ Faculty of Science, National Textile \\ University, Faisalabad, Punjab, Pakistan; \\ ${ }^{2}$ Faculty of Veterinary Science, \\ Institute of Pharmacy, Physiology, \\ and Pharmacology, University of \\ Agriculture, Faisalabad, Punjab, \\ Pakistan; ${ }^{3}$ Department of Computer \\ Science, COMSATS University, \\ Islamabad, Lahore Campus, Lahore, \\ Punjab, Pakistan; ${ }^{4}$ Department of \\ Information Technology, Government \\ College University, Faisalabad, Punjab, \\ Pakistan; ${ }^{5}$ Oncology Department, \\ Faisalabad Medical University, Allied \\ Hospital, Faisalabad, Punjab, Pakistan
}

This article was published in the following Dove Medical Press journal: Cancer Management and Research
Purpose: This retrospective study presents a comparative analysis of the overall survival and toxicities, as side effects, of docetaxel plus cyclophosphamide (TC) and doxorubicin plus cyclophosphamide (AC). The study measured their efficacies during adjuvant chemotherapy, treating Pakistani breast cancer patients by validating the results obtained, with the published analysis of the same treatment given to US patients.

Patients and methods: Between June 2015 and September 2017, for four chemotherapy cycles, 189 patients out of 358 received TC $\left(75 \mathrm{mg} / \mathrm{m}^{2}\right.$ of docetaxel, $600 \mathrm{mg} / \mathrm{m}^{2}$ of cyclophosphamide ) and 169 were treated with $\mathrm{AC}\left(60 \mathrm{mg} / \mathrm{m}^{2}\right.$ of doxorubicin, $600 \mathrm{mg} / \mathrm{m}^{2}$ of cyclophosphamide). On the basis of using pathological markers to assess patients, toxicities, as side effects, (due to docetaxel, doxorubicin, and cyclophosphamide) were listed in the database of this study. Common factors with respect to common terminology criteria for adverse events version 5.0 and side effects listed in MedlinePlus, NIH US database, and from the database of this study were then separated to be included in comparison for this study. Statistically, chi-squared test was used at $\alpha=0.05$.

Results: There was no statistically significant difference between the proportions of patients with vomiting, extreme tiredness, diarrhea, mild anemia, stability, and overall survival because $P$-value $>0.05$. However, AC remained less toxic $(P$-value $<0.05)$ by $22.6 \%, 25.7 \%, 25.3 \%$, $12.4 \%, 20.8 \%$, and $16.4 \%$ compared to TC for changes in taste, muscle pain, burning hands, change in hemoglobin level, moderate anemia, and needing blood transfusion respectively, whereas TC remained less toxic by $52.9 \%, 32.5 \%$, and $26.3 \%$ for dizziness, weight loss, and sores in throat and mouth, respectively.

Conclusion: At 27 months, TC was more toxic than AC, whereas both combinations had the same overall survival rate.

Keywords: patient health during chemotherapy, overall survival, TC vs AC, pathological markers, toxicity of anticancer drugs

\section{Introduction}

It is claimed that breast cancer is the most common ${ }^{1}$ and devastating disease. American Cancer Society estimated that there were 2,088,849 women suffering from breast cancer which were reported (in 2018) worldwide ${ }^{2}$ and 626,679 women died of this cancer. ${ }^{3}$ One study ${ }^{4}$ reported that among the cancer patients registered in the city of Lahore, Pakistan, from 2010 to 2012, 79\% were females who were suffering from breast cancer. 
Different anticancer drugs are used to treat different types of tumors ${ }^{5}$ with drug categories ${ }^{6}$ affecting abnormal cells in many ways. ${ }^{7}$ A chemotherapy schedule entails a treatment regime of anticancer drugs that are given for a set time duration at repeated intervals, called chemotherapy cycles. ${ }^{7-13}$ Chemotherapy may be given on different schedules depending upon two major aspects. The first aspect consists of patient factors including age, weight, and medical history. The second aspect consists of breast cancer factors including subtype, grade, stage, behavior (non-invasive or invasive), and disease history. In a drug schedule, a patient may receive the drug at repeated intervals, for example, once a week, once every 2 , 3 or 4 weeks. ${ }^{8,9,11,12,14}$ During the treatment phase, the patient may be given a single anticancer drug or a combination of different chemotherapy drugs simultaneously. ${ }^{7-12}$ For adjuvant treatment, researchers have shown that certain chemotherapy drug combinations are sometimes more effective than single drug treatment. ${ }^{11,15,16}$ To treat a breast tumor, chemotherapy drugs or their combinations may be given as adjuvant therapies such as doxorubicin plus cyclophosphamide (AC) and docetaxel plus cyclophosphamide (TC). ${ }^{7}$

Given the importance of knowing an anti-tumor drug's efficacy regarding side effects or infections, ${ }^{17}$ many studies have been completed and are being followed to compare different chemotherapies for cancer patients in the search for suitable drugs. ${ }^{16}$ Some drugs had high efficacy for some patients and others had comparatively high toxicity. There is a need to identify which drug had lower toxicity coupled with better tumor treatment in Pakistan as compared to other countries. To that end, the drug regime used for female breast cancer patients from a Pakistani Government Hospital in the period from June 2015 to September 2017 was studied. It was established that the following drug treatments were used: TC, AC, and epirubicin with the same combination. TC and AC combinations were then compared.

\section{Patients and methods Study design and patients}

In this retrospective study, female breast cancer patients from Allied Hospital in Pakistan, treated with adjuvant chemotherapy, were selected for the comparison of TC and AC during four cycles of both combinations. This study was approved by the ethical review committee of Faisalabad Medical University, Pakistan, approval number 675/2016, all patients provided written informed consent, and the study was conducted in accordance with the Declaration of Helsinki. The data of 782 cases of the patients suffering from breast tumors and who received or were receiving chemotherapy treatment were collected from their hematology reports from Allied Hospital Faisalabad. After applying the inclusion and exclusion criteria discussed in the following section, there were 489 cases to be studied. With initial screening, out of 489 patients, 6 male patients were removed from the samples. Out of 483 patients, 125 patients who were receiving treatment using drugs such as epirubicin, paclitaxel, and tamoxifen, were also separated from samples and excluded from the study. Thus, out of the remaining 358, there were 189 cases who were receiving or had been receiving adjuvant chemotherapy treatment of TC for four cycles and 169 female breast cancer patients were treated or being treated with AC (Figure 1).

\section{Inclusion criteria}

- Gender: female

- Age: $\geq 28$ and $\leq 62$ years

- Histology: invasive ductal carcinoma

- ECOG: 0-1

- Grade: all grades

- Stage: I-III

- Diabetes: non-diabetic

- Renal function tests (RFT): normal

- Ejection fraction (EF) in echocardiography: $55 \%-70 \%$

\section{Exclusion criteria}

- Gender: male

- Age: $\leq 28$ and $\geq 62$ years

- Histology: other than invasive ductal carcinoma

- ECOG: 2 and above

- Grade: all grades

- Stage: IV (metastasis)

- Diabetes: diabetic

- RFT: abnormal

- EF in echocardiography: $<50 \%$

Data from patients' admission charts and hematology followup reports were saved in the database of this study. With data organization of both cohorts done on the basis of common characteristics of TC and AC, different groups of ages, weights, and geographical areas were included. Figure 2 compares these groups of ages, areas, and weights of both TC and AC cohorts. A list of side effects was captured in a database which included the data of TC and AC combinations of the patients in the study. A separate list was made for the side effects of docetaxel, doxorubicin, and cyclophosphamide, given in MedlinePlus (a database of US National Library of Medicine). Figure 1 shows the common side effects taken from MedlinePlus and 


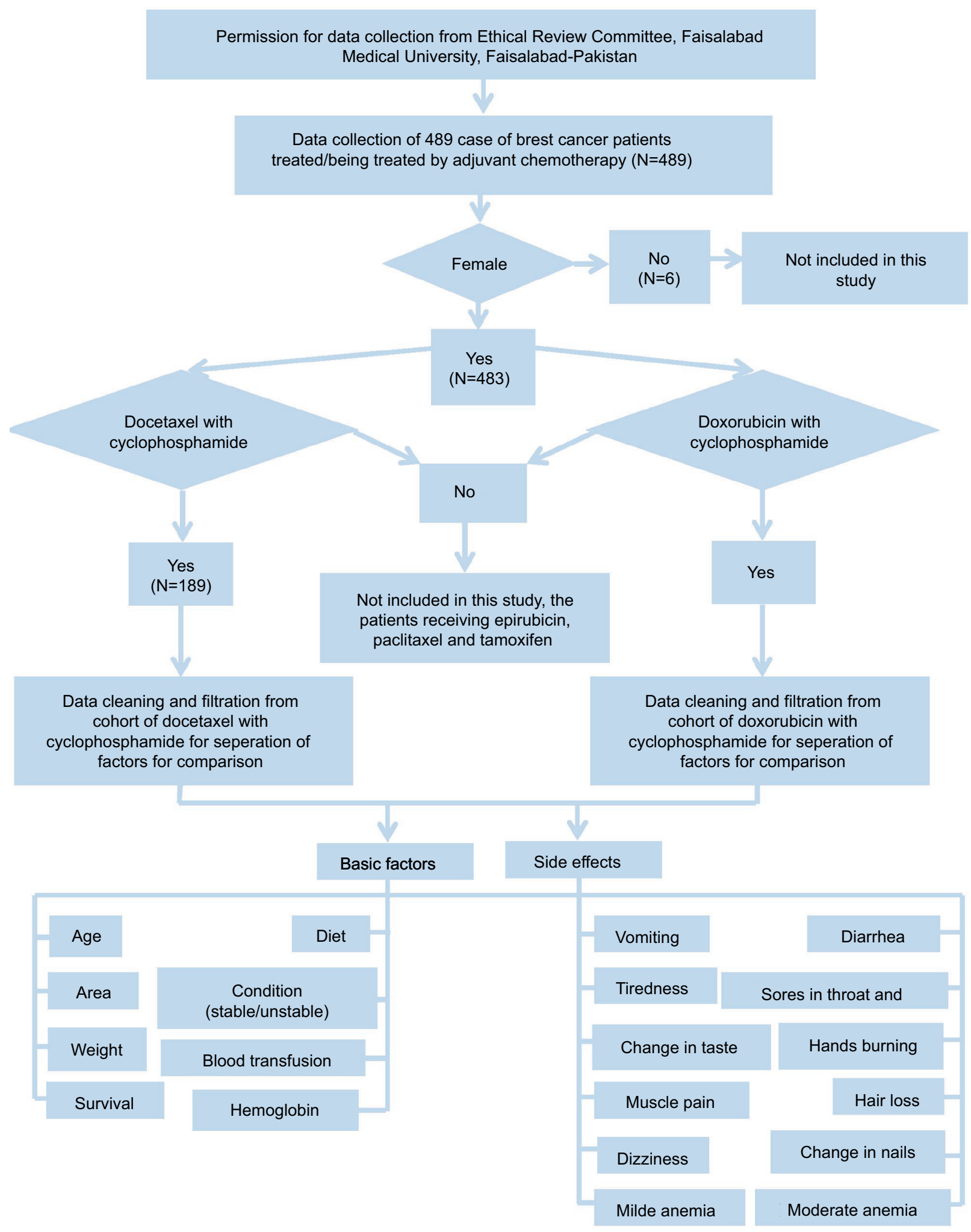

Figure I Flowchart of data collection, organization, filtration, and separation.

from the database of this study. The common characteristics of both cohorts were then divided into two common aspects including basic common factors and common side effects in order to compare the results of TC and AC on patients of these cohorts. Tables 1 and 2 show these basic factors and the common side effects with their respective attributes. 


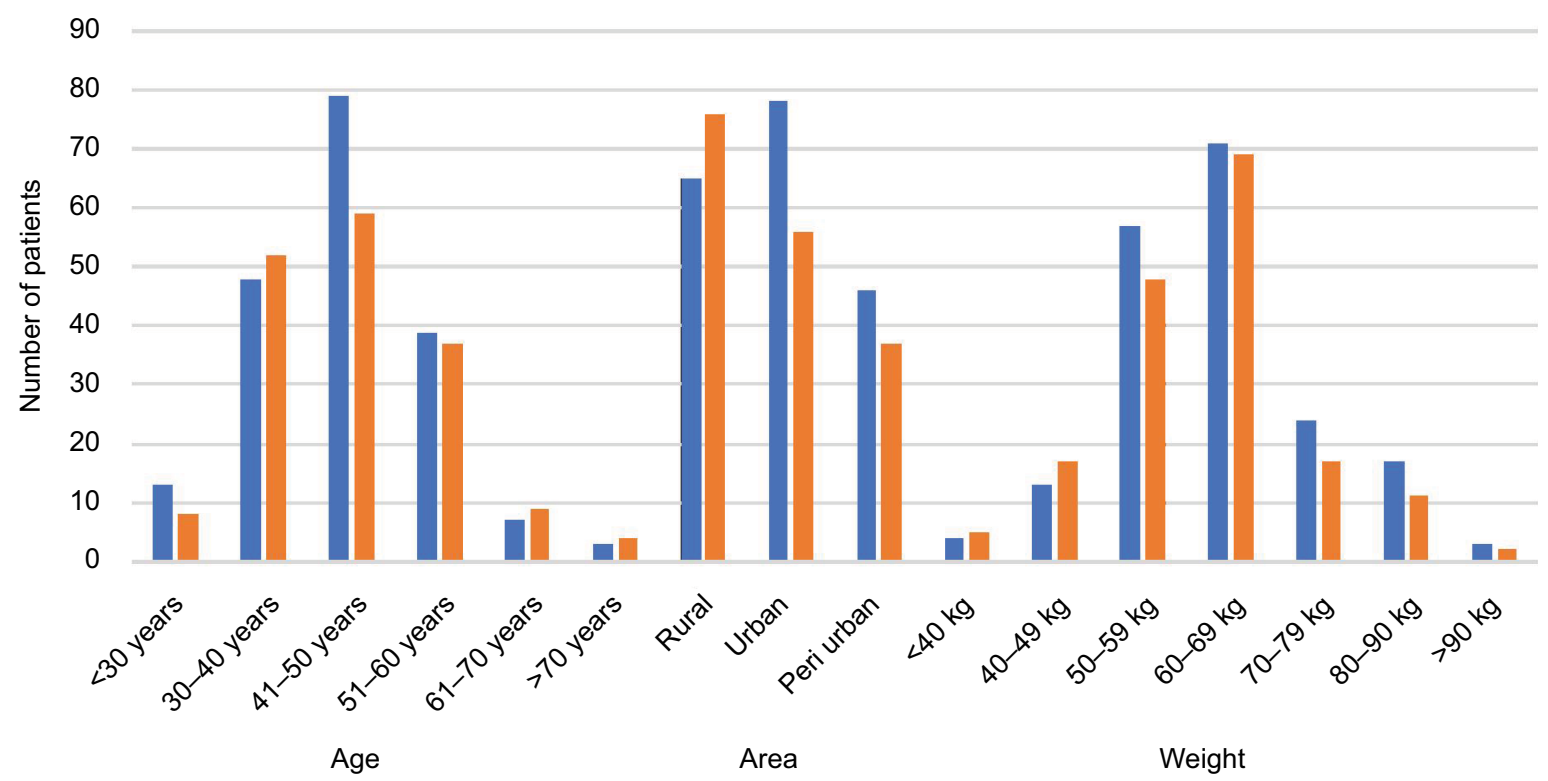

Figure 2 Number of female breast cancer patients receiving adjuvant chemotherapy based on age, area, and weight. Abbreviations: AC, doxorubicin plus cyclophosphamide; TC, docetaxel plus cyclophosphamide.

\section{Treatment}

A dosage, per cycle, of $75 \mathrm{mg}$ of docetaxel (intravenous) per $\mathrm{m}^{2}$ of patient body surface area, in combination with $600 \mathrm{mg} / \mathrm{m}^{2}$ cyclophosphamide was given in each of four cycles, one cycle consisting of 21 days. Similarly, varying from patient to patient, a dosage, per cycle, of $60 \mathrm{mg}$ of doxorubicin (intravenous) per $\mathrm{m}^{2}$ of patient body surface area, in combination with $600 \mathrm{mg} /$ $\mathrm{m}^{2}$ cyclophosphamide was given in each of four cycles. The patients with the same number of cycles were examined for the comparison of both TC and AC, observing their diet (good, moderate or poor) and their condition (stable or unstable).

\section{Aspects of TC and AC toxicity observed in this study (selected from US National Cancer Institute common terminology criteria for adverse events $[\mathrm{NCl}$ \\ CTCAE])}

With respect to US NCI CTCAE version 5.0, ${ }^{18}$ and MedlinePlus, US National Library of Medicine, ${ }^{19,20}$ the aspects for both the cohort of patients treated with TC (cpTC) and the cohort of patients treated with $\mathrm{AC}(\mathrm{cpAC})$ including hair loss, change of nail color, vomiting, extreme tiredness, changes in taste, muscle pain, diarrhea, sores in throat and mouth, burning in hands and feet, dizziness, moderate anemia, weight loss, stability, and mild anemia (Table 3), were studied. Other common factors that had changed in cpTC and cpAC were also observed including hemoglobin level, blood transfusion, and survival.

\section{The measured markers for cardiotoxicity and nephrotoxicity}

For cardiotoxicity ${ }^{21-24}$ in cpTC and cpAC, the values of echocardiographic ${ }^{25}$ measurements from one chemotherapy cycle to the following cycle (after every 21 days) were observed including peak gradient $(\mathrm{mmHg})$, mean gradient $(\mathrm{mmHg})$, mean gradient (diastolic), area of pressure half time (msec) (PHT) $\left(\mathrm{cm}^{2}\right)$, left ventricular outflow tract, regurgitation, regurgitation $\mathrm{PHT}$, regurgitant volume, $\mathrm{E} / \mathrm{A}$, and $\mathrm{E}$ wave deceleration time (msec) (DT) during four cycles of TC and similarly during the four cycles of AC.

For nephrotoxicity ${ }^{26}$ of both cpTC and cpAC, the changes in values of blood urea and serum creatinine (from their renal profile test reports), from one chemotherapy cycle to the following cycle (after every 21 days) were observed during four cycles of TC and similarly during the four cycles of AC.

\section{Statistical analysis}

Calculating the difference in overall trend in an individual patient's hemoglobin change because of n-cycles of chemotherapy

Lacking healthy red blood cells causes anemia, ${ }^{27}$ diagnosed by a complete blood count test. From the hemoglobin level, doctors decide the grade of anemia. Using NCI anemia 
Table I Basic factors with attributes used for comparison of TC and $A C$

\begin{tabular}{|c|c|}
\hline Factor & Attributes \\
\hline \multirow[t]{6}{*}{ Age (years) } & $<30$ \\
\hline & $30-40$ \\
\hline & $4 I-50$ \\
\hline & $5 I-60$ \\
\hline & $6 I-70$ \\
\hline & $>70$ \\
\hline \multirow[t]{3}{*}{ Area } & Rural \\
\hline & Urban \\
\hline & Peri-urban \\
\hline \multirow[t]{10}{*}{ Weight (kg) } & $<40$ \\
\hline & $40-49$ \\
\hline & $50-59$ \\
\hline & $60-69$ \\
\hline & 70-79 \\
\hline & $80-90$ \\
\hline & $>90$ \\
\hline & Stable \\
\hline & Loss \\
\hline & Gain \\
\hline \multirow[t]{2}{*}{ Survival } & Yes \\
\hline & No \\
\hline \multirow[t]{3}{*}{ Diet } & Good \\
\hline & Moderate \\
\hline & Poor \\
\hline \multirow[t]{2}{*}{ Stability } & Stable \\
\hline & Weak \\
\hline \multirow[t]{7}{*}{ Hemoglobin (g/dL) } & $>12$ \\
\hline & $11.5-11.9$ \\
\hline & $\mathrm{II}-1 \mathrm{I} .4$ \\
\hline & $10.5-10.9$ \\
\hline & $10-10.4$ \\
\hline & $9.5-9.9$ \\
\hline & $<9.5$ \\
\hline Blood transfusion & Yes \\
\hline
\end{tabular}

Abbreviations: AC, doxorubicin plus cyclophosphamide; TC, docetaxel plus cyclophosphamide.

scale, ${ }^{28}$ the hemoglobin level of patients of both TC and AC cohorts were compared to evaluate anemia presence resulting from chemotherapy of both combinations.

Given that patients in both TC and AC cohorts had four cycles, their hemoglobin levels were calculated. The overall trend of changing hemoglobin level during both TC and AC adjuvant chemotherapy (Figure 3) was also calculated. Equation 1 calculates the overall trend in patients' hemoglobin change, denoted by otHbc:

$$
o t H b c=\sum_{n}\left[\frac{\left(\frac{b H b-c H b n}{b H b}\right) \times 100}{n}\right]
$$

Table 2 Side effects with attributes used for comparison of TC and $A C$

\begin{tabular}{|c|c|}
\hline Side effect & Attributes \\
\hline \multirow[t]{2}{*}{ Vomiting } & Yes \\
\hline & No \\
\hline \multirow[t]{2}{*}{ Extreme tiredness or unusual weakness } & Yes \\
\hline & No \\
\hline \multirow[t]{2}{*}{ Changes in taste } & Yes \\
\hline & No \\
\hline \multirow[t]{2}{*}{ Muscle pain } & Yes \\
\hline & No \\
\hline \multirow[t]{2}{*}{ Diarrhea } & Yes \\
\hline & No \\
\hline \multirow[t]{2}{*}{ Sores in throat and mouth } & Yes \\
\hline & No \\
\hline \multirow[t]{2}{*}{ Burning in hands and feet } & Yes \\
\hline & No \\
\hline \multirow[t]{2}{*}{ Hair loss } & Yes \\
\hline & No \\
\hline \multirow[t]{2}{*}{ Change of nail color } & Yes \\
\hline & No \\
\hline \multirow[t]{2}{*}{ Dizziness } & Yes \\
\hline & No \\
\hline \multirow[t]{2}{*}{ Mild anemia } & Yes \\
\hline & No \\
\hline \multirow[t]{2}{*}{ Moderate anemia } & Yes \\
\hline & No \\
\hline
\end{tabular}

Abbreviations: AC, doxorubicin plus cyclophosphamide; TC, docetaxel plus cyclophosphamide.

where $\mathrm{bHb}$ is base hemoglobin value (with initial value) obtained before starting chemotherapy, $\mathrm{cHb}$ is current hemoglobin value obtained after the first cycle of chemotherapy, for example, before second, third, or fourth chemotherapy cycle, and $\mathrm{n}$ is from one to four cycles of chemotherapy.

\section{Calculating the difference in percentage value of a factor in both drug cohorts}

The difference in percentage value of the number of patients for both cohorts of a factor denoted by $D_{\text {factor }}$ was calculated by Equation 2:

$$
D_{\text {factor }}=p V_{1}-p V_{2}
$$

where $p V_{1}$ is percent value from one cohort and $p V_{2}$ is percent value from the second cohort of the same factor. $p V_{l}$ must be greater. In some factors, cpTC had greater value than cpAC and vice versa.

This study was designed to compare TC and AC as adjuvant chemotherapy treatment for female breast cancer patients on the basis of the presence or absence of 1-4 grade anemia (including mild, moderate, severe, and life-threatening), selected common factors, called routine physiological 
Table 3 Comparative analysis of TC and AC cohorts based on routine physiological parameters and common side effects

\begin{tabular}{|c|c|c|c|c|c|c|c|c|c|c|c|}
\hline \multirow[t]{2}{*}{ Parameter } & \multirow{2}{*}{\multicolumn{2}{|c|}{ Name }} & \multirow[b]{2}{*}{ Attribute } & \multicolumn{2}{|c|}{$\mathrm{TC}(\mathrm{N}=189)$} & \multicolumn{2}{|c|}{$A C(N=169)$} & \multicolumn{4}{|c|}{$\begin{array}{l}\text { Chi-squared test for homogeneity } \\
\text { of proportions at significance level: } \\
\alpha=0.05\end{array}$} \\
\hline & & & & Number & $\%$ & Number & $\%$ & $\chi^{2}$ & $d f$ & $\begin{array}{l}\text { Critical } \\
\text { value }\end{array}$ & $P$-value \\
\hline \multirow{16}{*}{$\begin{array}{l}\text { Common } \\
\text { factors }\end{array}$} & \multirow{2}{*}{\multicolumn{2}{|c|}{ Stability }} & Stable & 66 & 34.9 & 57 & 33.7 & \multirow[t]{2}{*}{0.056} & \multirow[t]{2}{*}{ I } & \multirow[t]{2}{*}{3.841} & \multirow[t]{2}{*}{0.812} \\
\hline & & & Weak & 123 & 65.1 & 112 & 66.3 & & & & \\
\hline & \multirow[t]{3}{*}{ Weight } & Loss & Yes & 64 & 33.8 & 112 & 66.3 & \multirow[t]{2}{*}{38.878} & \multirow[t]{2}{*}{1} & \multirow[t]{2}{*}{3.841} & \multirow[t]{2}{*}{0.000} \\
\hline & & & No & 123 & 66.1 & 54 & 32 & & & & \\
\hline & & \multicolumn{2}{|l|}{ Gain } & 2 & 1.1 & 3 & 1.7 & - & - & - & - \\
\hline & \multirow{7}{*}{\multicolumn{2}{|c|}{ Hemoglobin (g/dL) }} & $>12$ & 29 & 15.3 & 91 & 53.8 & \multirow[t]{7}{*}{83.931} & \multirow[t]{7}{*}{6} & \multirow[t]{7}{*}{12.592} & 0.000 \\
\hline & & & $11.5-11.9$ & 19 & 10.1 & 25 & 14.8 & & & & \\
\hline & & & $\mathrm{II}-\mathrm{II} .4$ & 38 & 20.1 & 27 & 16.0 & & & & \\
\hline & & & $10.5-10.9$ & 26 & 13.8 & 13 & 7.7 & & & & \\
\hline & & & $10-10.4$ & 28 & 14.8 & 6 & 3.6 & & & & \\
\hline & & & $9.5-9.9$ & 27 & 14.3 & 4 & 2.4 & & & & \\
\hline & & & $<9.5$ & 22 & 11.6 & 3 & 1.8 & & & & \\
\hline & Blood tra & sion & Yes & 59 & 31.2 & 25 & 14.8 & 13.402 & 1 & 3.841 & 0.000 \\
\hline & & & No & 130 & 68.8 & 144 & 85.2 & & & & \\
\hline & Survival & & Yes & 176 & 93.1 & 153 & 90.5 & 0.803 & 1 & 3.841 & 0.37 \\
\hline & & & No & 13 & 6.9 & 16 & 9.5 & & & & \\
\hline Side effects & Vomiting & & Yes & 177 & 93.3 & 149 & 88.2 & 3.298 & 1 & 3.841 & 0.069 \\
\hline & & & No & 12 & 6.3 & 20 & 11.8 & & & & \\
\hline & Extreme & Aness & Yes & 168 & 88.9 & 153 & 90.5 & 0.26 & I & 3.841 & 0.61 \\
\hline & & & No & 21 & 11.1 & 16 & 9.5 & & & & \\
\hline & Changes & & Yes & 55 & 29.1 & 11 & 6.5 & 30.283 & I & 3.841 & 0.000 \\
\hline & & & No & 134 & 70.9 & 158 & 93.5 & & & & \\
\hline & Muscle $\mathrm{p}$ & & Yes & 118 & 62.4 & 62 & 36.7 & 23.66 & I & 3.841 & 0.000 \\
\hline & & & No & 71 & 37.6 & 107 & 63.3 & & & & \\
\hline & Diarrhea & & Yes & 19 & 10.1 & 22 & 13 & 0.773 & I & 3.841 & 0.379 \\
\hline & & & No & 170 & 89.9 & 147 & 87 & & & & \\
\hline & Sores in & at and mouth & Yes & 127 & 67.2 & 158 & 93.5 & 38.003 & 1 & 3.841 & 0.000 \\
\hline & & & No & 62 & 32.8 & 11 & 6.5 & & & & \\
\hline & Burning $\mathrm{i}$ & nds and feet & Yes & 78 & 41.3 & 27 & 16 & 27.538 & 1 & 3.841 & 0.000 \\
\hline & & & No & 111 & 58.7 & 142 & 84 & & & & \\
\hline & Hair loss & & Yes & 189 & 100 & 169 & 100 & - & - & - & - \\
\hline & & & No & 0 & 0 & 0 & 0 & & & & \\
\hline & Dizziness & & Yes & 51 & 27 & 135 & 79.9 & 100.014 & I & 3.841 & 0.000 \\
\hline & & & No & 138 & 73 & 34 & 20.1 & & & & \\
\hline & Change $c$ & il color & Yes & 189 & 100 & 169 & 100 & - & - & - & - \\
\hline & & & No & 0 & 0 & 0 & 0 & & & & \\
\hline & Mild aner & & Yes & 105 & 55.6 & 92 & 54.4 & 0.045 & I & 3.841 & 0.832 \\
\hline & & & No & 84 & \begin{tabular}{|l|}
44.4 \\
\end{tabular} & 77 & 45.6 & & & & \\
\hline & Moderat & mia & Yes & 75 & 39.7 & 32 & 18.9 & 18.328 & 1 & $3.84 I$ & 0.000 \\
\hline & & & No & 114 & 60.3 & 137 & 81.1 & & & & \\
\hline
\end{tabular}

Abbreviations: AC, doxorubicin plus cyclophosphamide; TC, docetaxel plus cyclophosphamide.

parameters (including stability, hemoglobin, blood transfusion, and survival), and toxicity for selected and common side effects (including vomiting, extreme tiredness, changes in taste, muscle pain, diarrhea, sores in throat and mouth, burning in hands and feet, hair loss, dizziness, and change of nail color). In this study, through Minitab 17, chi-squared test for homogeneity of proportions (CTHP) at significance level $\alpha=0.05$ was used to compare these aspects. With the help of Equation 2 the difference was calculated by using percentage values. Using $\mathrm{R}$ programming language data, were organized and filtered along with computing values from Equations 1 and 2. 


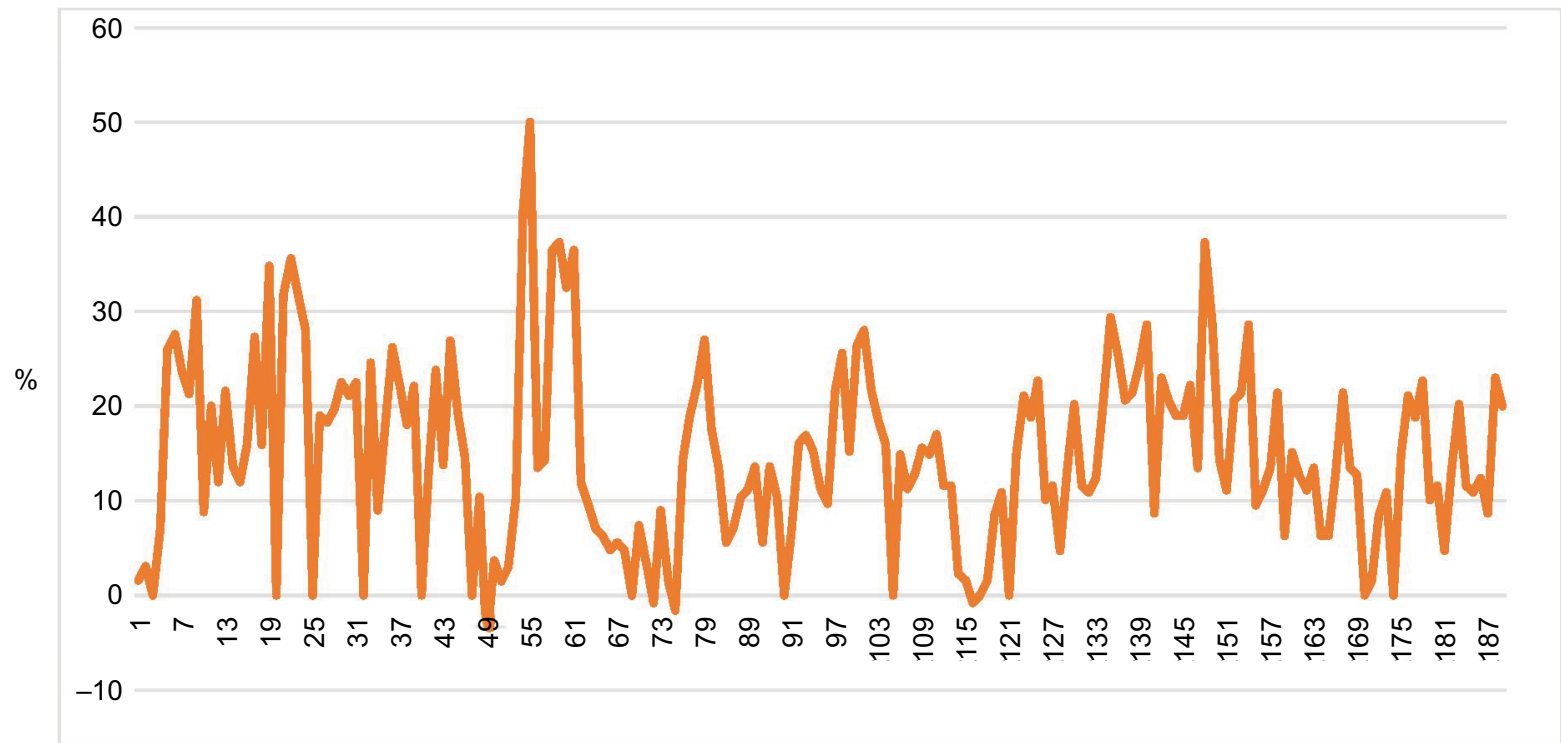

Percent change in patients' hemoglobin level caused by TC

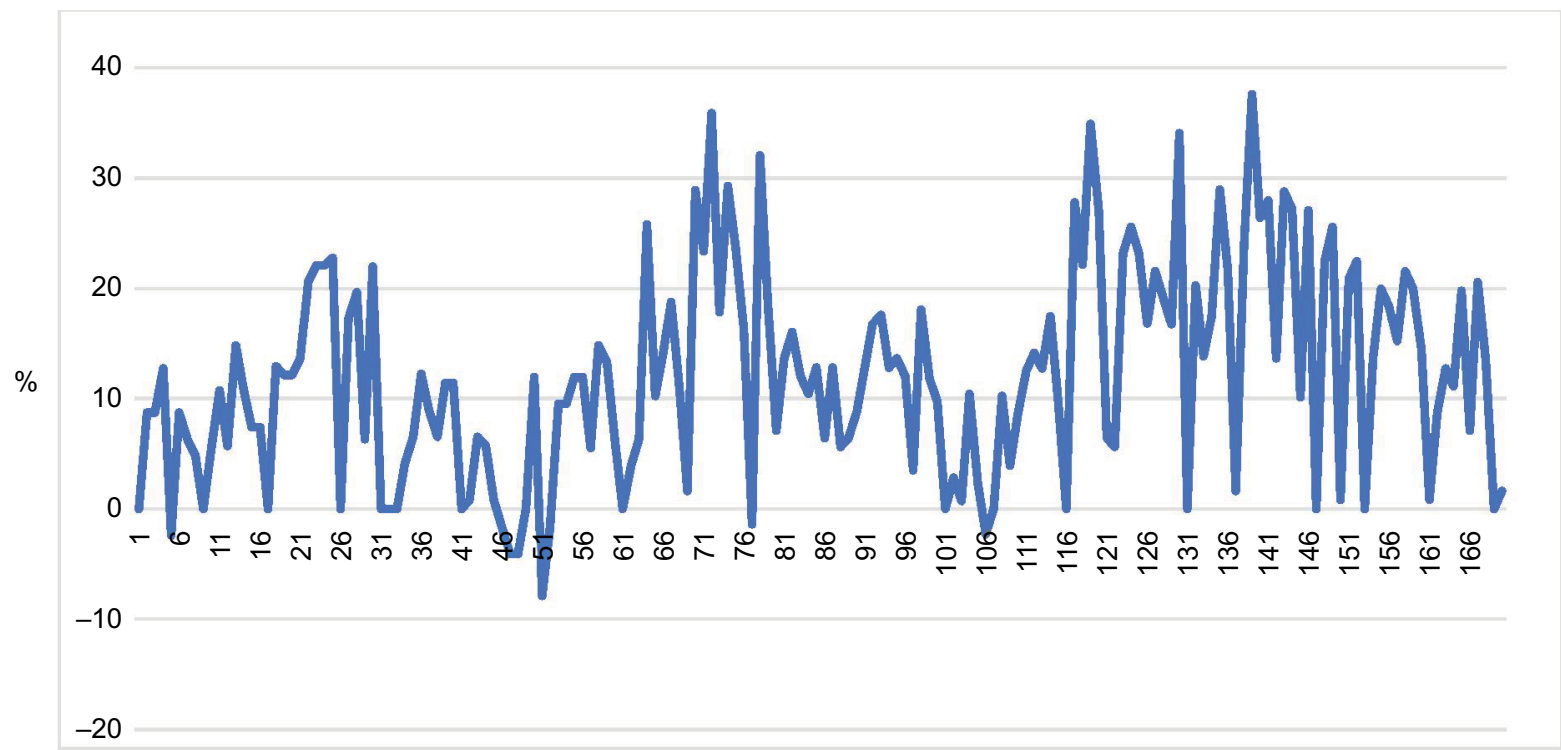

Percent change in patients' hemoglobin level caused by AC

Figure 3 Percent change in patients' hemoglobin level caused by adjuvant chemotherapy TC and AC drug combinations. Abbreviations: AC, doxorubicin plus cyclophosphamide; TC, docetaxel plus cyclophosphamide.

\section{Ethical approval}

All procedures performed in studies involving human participants were in accordance with the ethical standards of the institutional and/or national research committee. This study was approved by the ethical review committee of Faisalabad Medical University, approval number 675/2016, all patients provided written informed consent, and the study was conducted in accordance with the Declaration of Helsinki.

\section{Results}

\section{Aspects of comparison}

This study included four common factors (routine physiological parameters) of both cpTC and cpAC, listed in Table 3 . 
The results of this study show common side effects, of both combinations, in which two (hair loss and change of nail color) were not listed in the comparison of cpTC and cpAC because these were common to all patients being studied. The results for the remaining eleven side effects are given in Table 3.

\section{Cardiotoxicity}

Evaluating echocardiography of the cpTC and cpAC, it was observed that all the echocardiographic ${ }^{25}$ measurements remained normal for all patients treated with both TC and AC (Table 4), whereas EF of most of the patients treated with $\mathrm{AC}$ remained a bit low.

\section{Nephrotoxicity}

Evaluating renal profiles of the cpTC and cpAC, it was observed that the value of blood urea of the cpTC was 21-36 $\mathrm{mg} / \mathrm{dL}$, and thus remained in its normal value $(10-45 \mathrm{mg}$ / $\mathrm{dL}$ ). During four cycles, in cpTC, it was also noted that there was a minor difference in the value of blood urea of a patient from one cycle to the following cycle of TC. Similarly, the value of blood urea of the cpAC was $18-33 \mathrm{mg} / \mathrm{dL}$, and thus remained in its normal value $(10-45 \mathrm{mg} / \mathrm{dL})$. During four cycles, in cpAC, it was also noted that there was a minor difference in the value of blood urea of a patient from one cycle to the following cycle of AC.

In renal profiles of the cpTC and cpAC, it was observed that the value of serum creatinine of the cpTC was $0.6-1$ $\mathrm{mg} / \mathrm{dL}$, and thus remained in its normal value $(0.6-1.2 \mathrm{mg} /$ $\mathrm{dL})$. During four cycles, in cpTC, it was also noted that there was a minor difference in the value of serum creatinine of a patient from one cycle to the following cycle of TC. Similarly, the value of serum creatinine of the cpAC was $0.6-1.1 \mathrm{mg} /$ $\mathrm{dL}$, and thus remained in its normal value $(0.6-1.2 \mathrm{mg} / \mathrm{dL})$.
During four cycles, in cpAC, it was also noted that there was a minor difference in the value of serum creatinine of a patient from one cycle to the following cycle of AC.

The aforementioned results of two major renal profiles (which contributed to determining nephrotoxicity) showed that, for both cpTC and cpAC, they remained normal, therefore both TC and AC were not found to be nephrotoxic to patients in this study.

\section{Vomiting}

By applying CTHP, the proportions of vomiting patients who were treated with both TC (177 out of 189 patients, 93.3\%) and AC (149 out of 169 patients, 88.2\%) were obtained, and since $P=0.069>0.05$, there was no difference in the proportions of vomiting patients treated with either TC or AC.

\section{Extreme tiredness}

There were proportions of patients with extreme tiredness who were treated with both TC (88.9\%) and AC (90.5\%), and since $P=0.61>0.05$, there was no difference in the proportions of patients who experienced extreme tiredness because of treatment with either TC or AC.

\section{Changes in taste}

After applying CTHP on proportions of patients who had a change in taste, who were treated with both combinations, TC (29.1\%) and $\mathrm{AC}(6.5 \%)$, since $P=0<0.05$, there was a significant difference in their proportions. Of patients who experienced changes in taste, $22.6 \%$ fewer were treated with AC than with TC.

\section{Muscle pain}

In proportions of patients who experienced muscle pain, who were treated with both TC (62.4\%) and AC (36.7\%), since $P=0<0.05$, there was a significant difference in their

Table 4 Echocardiographic measurements of patients treated with TC and AC

\begin{tabular}{|c|c|c|c|}
\hline Measurement & Normal value & TC & AC \\
\hline Peak gradient $(\mathrm{mmHg})$ & $26-36 \mathrm{~mm}$ & $27-31$ & $26-32$ \\
\hline Mean gradient $(\mathrm{mmHg})$ & $19-39 \mathrm{~mm}$ & $27-32$ & $27-33$ \\
\hline Mean gradient (diastolic) & $08-12 \mathrm{~mm}$ & $9-10$ & $9-10$ \\
\hline Area of PHT $\left(\mathrm{cm}^{2}\right)$ & $07-11 \mathrm{~mm}$ & $8-9$ & $8-9$ \\
\hline LVOT & $36-56 \mathrm{~mm}$ & $42-49$ & $42-51$ \\
\hline Regurgitation & $25-41 \mathrm{~mm}$ & $28-32$ & $27-33$ \\
\hline EF & $50 \%-65 \%$ & $55-60$ & $48-56$ \\
\hline Regurgitant volume & $29 \%-37 \%$ & $31-35$ & $30-36$ \\
\hline E/A & $\mathrm{I}-\mathrm{I} .5$ & $1-1.18$ & $1-1.2$ \\
\hline DT & $160-240 \mathrm{~ms}$ & $198-220$ & $192-228$ \\
\hline
\end{tabular}

Abbreviations: AC, doxorubicin plus cyclophosphamide; DT, E wave deceleration time (msec); E/A, the ratio of peak velocity blood flow from gravity in early diastole (the E wave) to peak velocity flow in late diastole caused by atrial contraction (the A wave); EF, ejection fraction; LVOT, left ventricular outflow tract; PHT, pressure half time (msec); TC, docetaxel plus cyclophosphamide. 
proportions. Of patients who suffered from muscle pain, $25.7 \%$ fewer were treated with AC than with TC.

\section{Diarrhea}

In proportions of patients who suffered from diarrhea, who were treated with both TC (10.1\%) and AC (13\%), since $P=0.379>0.05$, there was no difference in the proportions of patients who suffered from diarrhea because of treatment with either TC or AC.

\section{Sores in throat and mouth}

In proportions of patients who experienced sores in throat and mouth, who were treated with both TC (67.2\%) and AC (93.5\%), since $P=0<0.05$, there was a significant difference in their proportions. Of patients who experienced sores in throat and mouth, $26.3 \%$ fewer were treated with TC than with AC.

\section{Burning in hands and feet}

In proportions of patients who experienced burning in hands and feet, who were treated with both TC (41.3\%) and AC (16\%), since $P=0<0.05$, there was a significant difference in their proportions. Of patients who experienced burning in hands and feet, $25.3 \%$ fewer were treated with $\mathrm{AC}$ than with TC.

\section{Dizziness}

In proportions of patients experiencing dizziness, who were treated with both TC (27\%) and AC (79.9\%), since $P=0<0.05$, there was a significant difference in their proportions. Of patients who experienced dizziness, $52.9 \%$ fewer were treated with TC than with AC.

\section{Moderate anemia}

In proportions of moderate anemia in patients who were treated with both TC (39.7\%) and AC (18.9\%), since $P=0<0.05$, there was a significant difference in their proportions. Of patients who suffered from moderate anemia, 20.8\% fewer were treated with AC than with TC.

\section{Weight loss}

In proportions of patients who lost weight who were treated with both TC $(33.8 \%)$ and $\mathrm{AC}(66.3 \%)$, since $P=0<0.05$, there was a significant difference in their proportions. Of patients who lost weight, $32.5 \%$ fewer were treated with TC than with AC. A proportion of $1.1 \%$ and $1.7 \%$ patients gained weight, treated with $\mathrm{TC}$ and $\mathrm{AC}$, respectively.

\section{Stability}

In proportions of stability in patients who were treated with both TC (34.9\%) and $\mathrm{AC}(33.7 \%)$, since $P=0.812>0.05$, there was no difference in proportions of stability in patients treated with either TC or AC.

\section{Mild anemia}

In proportions of mild anemia in patients who were treated with both TC (55.6\%) and AC (54.4\%), since $P=0.832>0.05$, there was no difference in proportions of mild anemia in patients treated with either TC or AC.

\section{Blood transfusion}

In proportions of patients to who received blood transfusion during treatment with both TC (31.2\%) and AC (14.8\%), since $P=0<0.05$, there was a significant difference in their proportions. Blood was transferred to $16.4 \%$ fewer patients treated with AC than with TC.

\section{Hemoglobin level}

In proportions of patients' hemoglobin level, during treatment with TC $(>12 \mathrm{~g} / \mathrm{dL}=15.3 \%, 11.5-11.9 \mathrm{~g} / \mathrm{dL}$ $=10.1 \%, 11-11.4 \mathrm{~g} / \mathrm{dL}=20.1 \%, 10.5-10.9 \mathrm{~g} / \mathrm{dL}=13.8 \%$, $10-10.4 \mathrm{~g} / \mathrm{dL}=14.8 \%, 9.5-9.9 \mathrm{~g} / \mathrm{dL}=14.3 \%,<9.5 \mathrm{~g} /$ $\mathrm{dL}=11.6 \%)$ and $\mathrm{AC}(>12 \mathrm{~g} / \mathrm{dL}=53.8 \%, 11.5-11.9 \mathrm{~g} / \mathrm{dL}$ $=14.8 \%, 11-11.4 \mathrm{~g} / \mathrm{dL}=16.0 \%, 10.5-10.9 \mathrm{~g} / \mathrm{dL}=7.7 \%$, $10-10.4 \mathrm{~g} / \mathrm{dL}=3.6 \%, 9.5-9.9 \mathrm{~g} / \mathrm{dL}=2.4 \%,<9.5 \mathrm{~g} / \mathrm{dL}$ $=1.8 \%$ ), since $P=0.000<0.05$, there was a significant difference in their proportions. A lower change of $12.35 \%$ in overall difference of hemoglobin level was calculated by Equations 1 and 2, for patients who were treated with $\mathrm{AC}$ than with TC.

\section{Survival}

In proportions of patients' survival treated with both TC (93.1\%) and $\mathrm{AC}(90.5 \%)$, since $P=0.37>0.05$, there was no difference in proportions of patients' survival treated with either TC or AC.

\section{Discussion}

Different studies were presented including disease related to the health status and toxicity of anticancer drugs for breast cancer patients. ${ }^{13,29,30}$ There are different combinations of chemotherapy for postoperative therapy in breast cancer, ${ }^{31}$ especially combinations of docetaxel with doxorubicin and doxorubicin with cyclophosphamide as the first line of chemotherapy in invasive breast cancer. ${ }^{32}$ Another comparison was presented for different combinations including AC followed by docetaxel after a cycle of 21 days with doxorubicin and docetaxel every 14 days as neoadjuvant chemotherapy in operable breast cancer ${ }^{33}$ that involve results of a multicenter, randomized, phase III trial. ${ }^{34}$ 
For validation, the results of this study were compared with published results ${ }^{32}$ which found TC, with a different toxicity profile, more superior in terms of disease-free survival than $\mathrm{AC}$, whereas this study found $\mathrm{AC}$ was less toxic than TC in more aspects. The TC cohort of grade 1, 2, 3, and 4 under their study suffered from asthenia with percentages of $43,32,3$, and $<1$, respectively, a total of $78 \%$ compared to the AC cohort of all grades who suffered at percentages of $42,31,4$, and $<1$, respectively, a total of $77 \%$. Whereas in this study, $88.9 \%$ of the TC cohort of all grades suffered from asthenia compared with $90.5 \%$ of the AC cohort of all grades.

The TC cohort of all grades in their study suffered from myalgia with percentages of $22,10,1$, and $<1$, respectively, a total of $33 \%$, compared to the AC cohort of all grades who suffered at percentages of $11,5,1$, and $<1$, respectively, a total of $17 \%$. Whereas in this study, $62.4 \%$ of the TC cohort of all grades suffered from myalgia compared to $36.7 \%$ of the AC cohort of all grades.

The TC cohort of all grades in their study suffered from stomatitis with percentages of $23,10,1$, and $<1$, respectively, a total of $34 \%$, compared to the AC cohort of all grades who suffered at percentages of $29,15,1$, and $<1$, respectively, a total of $46 \%$. Whereas in this study, $67.2 \%$ of the TC cohort of all grades suffered from stomatitis compared with $93.5 \%$ of the AC cohort of all grades.

The TC cohort of all grades in their study suffered from vomiting with percentages of $9,5,1$ and $<1$, respectively, a total of $17 \%$, compared with the AC cohort of all grades who suffered at percentages of $21,16,5$, and $<1$, respectively, a total of $42 \%$. Whereas $93.3 \%$ of the TC cohort of all grades suffered from vomiting in this study compared with $88.2 \%$ of the AC cohort of all grades.

The TC cohort of all grades in their study suffered from nausea with percentages of $38,13,2$, and $<1$, respectively, a total of $53 \%$, compared with the AC cohort of all grades who suffered at percentages of $43,32,7$, and 1 , respectively, a total of $82 \%$. Whereas $10 \%$ of the TC cohort of all grades in this study suffered from nausea compared to $13 \%$ of the AC cohort of all grades.

TC cohort of all grades in their study suffered from infection with percentages of $8,4,7$, and $<1$, respectively, a total of $19 \%$, compared with the AC cohort of all grades who suffered at percentages of $7,5,8$, and $<1$, respectively, a total of $20 \%$. Whereas $41 \%$ of the TC cohort of all grades in this study suffered from infection compared to $16 \%$ of the AC cohort of all grades.
The TC cohort of all grades in their study suffered from anemia with percentages of $3,2,1$, and $<1$, respectively, a total of $6.8 \%$, compared with the AC cohort of all grades who suffered at percentages of $4,3,1$, and $<1$, respectively, a total of $8 \%$. Whereas in this study, $55.6 \%$ of the TC cohort of all grades suffered from anemia compared with $54.4 \%$ of the AC cohort of all grades.

Jones et $\mathrm{al}^{32}$ found that the toxicities in general, were fairly similar between TC and AC groups but with certain exceptions. TC cohort faced significantly more grade 1 and 2 myalgia, edema, and arthralgia, whereas AC cohort with grade 1-4 had more vomiting and nausea. They also observed that in AC cohort, a single patient expired because of congestive heart failure, and four patients passed away because of myocardial infarction. In TC cohort, not a single case of congestive heart failure was seen; however, two patients expired because of myocardial infarction. Greater levels of neutropenia and fever were seen with TC in 25 patients, compared with AC (13 patients). Two patients passed away (one death due to neutropenia and sepsis and one unrelated cardiac death) while being treated with TC; not a single patient expired while being treated with AC. They found clinically significant toxicities, as discussed previously. No cases of myelodysplasia or leukemia were observed.

\section{Conclusion}

It has been observed that $\mathrm{TC}$ is less toxic across the aspects of TC and AC toxicity studied in this work. No statistically significant difference in the proportions of patients treated with both TC and AC combinations with regards to vomiting, extreme tiredness, diarrhea, stability, mild anemia, and overall survival was found. However, TC was less toxic in terms of weight loss, dizziness, and sores in throat and mouth in comparison with AC (Figure 4), whereas AC was less toxic in terms of change in taste, muscle pain, burning in hands and feet, change in hemoglobin level, moderate anemia, and needing blood transfusion compared to TC. Therefore, it is concluded that doxorubicin with cyclophosphamide may give better results in stopping tumor growth with lower toxicity, as mentioned in Table 2. Hemoglobin level, weight loss and gain, and blood transfusion in patients treated with TC and $\mathrm{AC}$ were the novel aspects compared in this study.

\section{Acknowledgments}

Dr Khawar Nadeem, his staff at the Oncology Department, and Mr Jawad Ahmad, IT Department, Allied Hospital, 


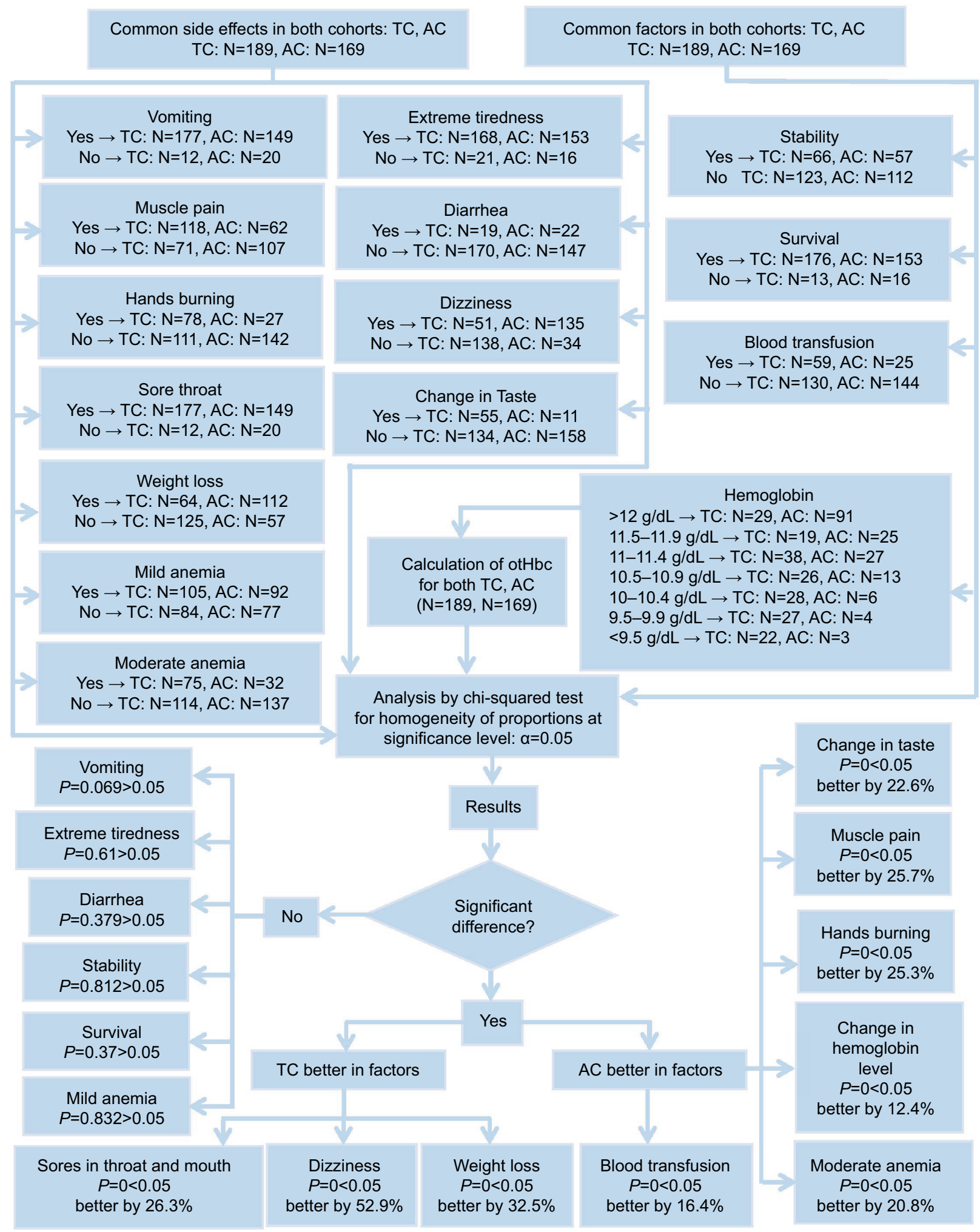

Figure $4 \mathrm{~A}$ summary of the analytical results.

Abbreviations: AC, doxorubicin plus cyclophosphamide; otHbc, overall trend in patients' hemoglobin change; TC, docetaxel plus cyclophosphamide. 
Faisalabad, are acknowledged for helping with the data collection in this study.

\section{Author contributions}

All authors contributed to data analysis, drafting or revising the article, gave final approval of the version to be published, and agree to be accountable for all aspects of the work.

\section{Disclosure}

The authors report no conflicts of interest in this work.

\section{References}

1. Miller KD, Siegel RL, Lin CC, et al. Cancer treatment and survivorship statistics, 2016. CA Cancer J Clin. 2016;66(4):271-289.

2. Bray F, Ferlay J, Soerjomataram I, et al. Global cancer statistics 2018 : GLOBOCAN estimates of incidence and mortality worldwide for 36 cancers in 185 countries. CA Cancer J Clin. 2018;68(6):394-424.

3. Ferlay J, Soerjomataram I, Dikshit R, et al. Cancer incidence and mortality worldwide: sources, methods and major patterns in GLOBOCAN 2012. Int J Cancer. 2015;136(5):E359-E386.

4. Badar F, Mahmood S. Epidemiology of cancers in Lahore, Pakistan, among children, adolescents and adults, 2010-2012: a cross-sectional study part 2. BMJ Open. 2017;7(12):e016559.

5. Desantis CE, Lin CC, Mariotto AB, et al. Cancer treatment and survivorship statistics, 2014. CA Cancer J Clin. 2014;64(4):252-271.

6. Teicher BA. Anticancer Drug Development Guide: Preclinical Screening, Clinical Trials, and Approval. 2nd ed. Germany: Springer Science \& Business Media; 2013.

7. Pratt WB. The Anticancer Drugs. 1994 ed. USA: Oxford University Press; 1994.

8. Senkus E, Kyriakides S, Ohno S, et al. Primary breast cancer: ESMO Clinical Practice Guidelines for diagnosis, treatment and follow-up. Ann Oncol. 2015;26(suppl 5):v8-v30.

9. Gradishar WJ, Anderson BO, Balassanian R, et al. Invasive breast cancer version 1.2016, NCCN clinical practice guidelines in oncology. $J$ Natl Compr Canc Netw. 2016;14(3):324-354.

10. Gradishar W, Anderson B, Balassanian R. NCCN Clinical Practice Guidelines in Oncology: Breast Cancer, version 1.2017; 2017. Available from: https://wwwnccnorg/store/login/loginaspx?ReturnURL=https:// wwwnccnorg/professionals/physician_gls/pdf/breastpdf. Accessed December 17, 2018.

11. Gradishar WJ, Anderson BO, Balassanian R, et al. NCCN Guidelines Insights: Breast Cancer, Version 1.2017. J Natl Compr Canc Netw. 2017;15(4):433-451.

12. Runowicz CD, Leach CR, Henry NL, et al. American cancer society/ American society of clinical oncology breast cancer survivorship care guideline. CA Cancer J Clin. 2016;66(1):43-73.

13. Hoffmann T, Corrêa-Fissmer M, Duarte CS, Nazário RF, Barranco ABS, Oliveira KWK. Prevalence of dermatological complaints in patients undergoing treatment for breast cancer. An Bras Dermatol. 2018;93(3):362-367.

14. Hugh J, Hanson J, Cheang MC, et al. Breast cancer subtypes and response to docetaxel in node-positive breast cancer: use of an immunohistochemical definition in the BCIRG 001 trial. J Clin Oncol. 2009;27(8):1168-1176.

15. Kumar S, Choudhary S, Singh N, Setty M. Combination of natural drugs: an emerging trend in cancer chemotherapy. J Drug Deliv Ther. 2012;2(3):97-105.

16. Li S, Yuan S, Zhao Q, Wang B, Wang X, Li K. Quercetin enhances chemotherapeutic effect of doxorubicin against human breast cancer cells while reducing toxic side effects of it. Biomed Pharmacother. 2018;100:441-447.
17. Tang J, Zhang R, Guo M, et al. Nucleosome-inspired nanocarrier obtains encapsulation efficiency enhancement and side effects reduction in chemotherapy by using fullerenol assembled with doxorubicin. Biomaterials. 2018;167:205-215.

18. US-NCI. CTCAE v5.0 Clean, Tracked, and Mapping Document (Excel); 2017. Available from: https://ctep.cancer.gov/protocolDevelopment/ electronic_applications/ctc.htm\#ctc_50. Accessed October 07, 2018.

19. The American Society of Health-System Pharmacists I. Doxorubicin; 2012. Available from: https://medlineplus.gov/druginfo/meds/a682221. html. Accessed October 07, 2018.

20. The American Society of Health-System Pharmacists I. Docetaxel. 2014; 2018. Available from: https://medlineplus.gov/druginfo/meds/ a696031.html. Accessed October 07, 2018.

21. Frères $P$, Bouznad N, Servais L, et al. Variations of circulating cardiac biomarkers during and after anthracycline-containing chemotherapy in breast cancer patients. BMC Cancer. 2018;18(1):102.

22. Dolci A, Dominici R, Cardinale D, Sandri MT, Panteghini M. Biochemical markers for prediction of chemotherapy-induced cardiotoxicity: systematic review of the literature and recommendations for use. $\mathrm{Am} J$ Clin Pathol. 2008;130(5):688-695.

23. Chan S, Friedrichs K, Noel D, et al. Prospective randomized trial of docetaxel versus doxorubicin in patients with metastatic breast cancer. J Clin Oncol. 1999;17(8):2341-2354.

24. Thavendiranathan P, Poulin F, Lim KD, Plana JC, Woo A, Marwick $\mathrm{TH}$. Use of myocardial strain imaging by echocardiography for the early detection of cardiotoxicity in patients during and after cancer chemotherapy: a systematic review. J Am Coll Cardiol. 2014;63(25 Pt A):2751-2768.

25. Schvartzman PR, Fuchs FD, Mello AG, Coli M, Schvartzman M, Moreira LB. Normal values of echocardiographic measurements. A population-based study. Arq Bras Cardiol. 2000;75(2):111-114.

26. Mohri J, Katada C, Ueda M, et al. Predisposing factors for chemotherapy-induced nephrotoxicity in patients with advanced esophageal cancer who received combination chemotherapy with docetaxel, cisplatin, and 5-fluorouracil. J Transl Int Med. 2018;6(1):32-37.

27. Beutler E, Waalen J. The definition of anemia: what is the lower limit of normal of the blood hemoglobin concentration? Blood. 2006;107(5):1747-1750.

28. US-Department-of-Health-and-Human-Services. Common Terminology Criteria for Adverse Events (CTCAE) version 4.0: National Cancer Institute; 2009:09-5410.

29. Martín M, Lluch A, Seguí MA, et al. Toxicity and health-related quality of life in breast cancer patients receiving adjuvant docetaxel, doxorubicin, cyclophosphamide (TAC) or 5-fluorouracil, doxorubicin and cyclophosphamide (FAC): impact of adding primary prophylactic granulocyte-colony stimulating factor to the TAC regimen. Ann Oncol. 2006;17(8):1205-1212.

30. Bayo J, Aviñó V, Toscano F, Jiménez F. Toxicity of docetaxel, carboplatin, and trastuzumab combination as adjuvant or neo-adjuvant treatment for Her2 positive breast cancer patients and impact of colony-stimulating factor prophylaxis. Breast J. 2018;24(4):462-467.

31. Bonadonna G, Brusamolino E, Valagussa P, et al. Combination chemotherapy as an adjuvant treatment in operable breast cancer. $N$ Engl J Med. 1976;294(8):405-410.

32. Jones SE, Savin MA, Holmes FA, et al. Phase III trial comparing doxorubicin plus cyclophosphamide with docetaxel plus cyclophosphamide as adjuvant therapy for operable breast cancer. J Clin Oncol. 2006;24(34):5381-5387.

33. von Minckwitz G, Raab G, Caputo A, et al. Doxorubicin with cyclophosphamide followed by docetaxel every 21 days compared with doxorubicin and docetaxel every 14 days as preoperative treatment in operable breast cancer: the GEPARDUO study of the German Breast Group. J Clin Oncol. 2005;23(12):2676-2685.

34. Nabholtz JM, Falkson C, Campos D, et al. Docetaxel and doxorubicin compared with doxorubicin and cyclophosphamide as first-line chemotherapy for metastatic breast cancer: results of a randomized, multicenter, phase III trial. J Clin Oncol. 2003;21(6):968-975. 
Cancer Management and Research

\section{Publish your work in this journal}

Cancer Management and Research is an international, peer-reviewed open access journal focusing on cancer research and the optimal use of preventative and integrated treatment interventions to achieve improved outcomes, enhanced survival and quality of life for the cancer patient.

The manuscript management system is completely online and includes

Submit your manuscript here: https://www.dovepress.com/cancer-management-and-research-journal 